\title{
COMPRESSIVE SENSING BASED SIGNAL RECOVERY WITH DIFFERENT TRANSFORMS
}

\author{
DR. MOHAMMED AHMED SHAKIR \\ Dept. of Electrical and Computer,Collage of Engineering, University of Duhok, Kurdistan Region-Iraq
}

\begin{abstract}
Compressive sensing (CS) is a new technique that give an approach to reconstruct the signal with few numbers of observations or measurements. The CS is based on $L_{1}$-norm minimizations to find the sparse solutions and it is known as basis pursuit.

In this investigation, CS scheme with different transforms is proposed. The three utilized transform techniques are: Discrete Fourier Transform(DFT), Discrete Cosine Transform(DCT) and Discrete Wavelet Transform(DWT) with daubechies1(DB1) and coiflets1(coif1) basis. The proposed system is tested by employing the following signals: Blocks, Heavy Sine, 'Bumps' and 'Doppler' which cover wide range of applications. The four testing signals are represented in sparse domain using different transforms. In order to threshold the coefficients of the signals in sparse domain, the universal threshold is utilized in the case of CS with FFT and DWT whereas, the universal threshold is modified to prune the DCT coefficients.

The main aim of this study is to investigate the differences among CS with DFT, CS with DCT and CS with DWT, and consequently a suitable transform domain used with CS to be selected. The comparative study is established by assessing the performance of the proposed system using Root Mean Square Error (RMSE), output SNR, and the time required to reconstruct approximated signals.

Simulation results have shown that the CS with DWT outperforms the CS with FFT and DCT. CS with DWT has achieved good RMSE values about $(0.0014$ to $3.359 \mathrm{e}-8)$ even when half of the signal elements are removed. CS with FFT and DCT enhanced the noisy Blocks and Bumps signals by 3dB and 1dB respectively, while it is failed to enhance noisy Heavy Sine and Doppler signals. CS with DWT of two basis and for single decomposition level have improved the noisy Blocks, Bumps, Heavy Sine and Doppler signals by 5dB, 4dB, $3 \mathrm{~dB}$, and $3 \mathrm{~dB}$ respectively.
\end{abstract}

KEYWORDS: Compressive Sensing, Sparsity, DCT, DFT, DWT, L1-Norm.

\section{1- INTRODUCTION}

Tn the period of digital technology innovation, the data acquisition/sensing instruments are producing enormous amount of data that should be transmitted, processed, or stored. This is a popular issue in sensing systems dealing with multimedia signals, medical and biomedical data, radar signals, and so forth. The required amount of digital information given as the number of digital samples, measurements or observations per time instance is determined based on Shannon-Nyquist sampling theorem. Consequently, a signal can be recovered if the sampling frequency $\left(F_{S}\right)$ is at least greater than two times the maximum frequency contained in the signal $\left(2 f_{\max }\right)$. Clearly, the sampling technique results in a large number of samples for signals with significantly high maximal frequency [1].

The signal acquisition process in the real applications was mainly done as stated by the Nyquist rate. To respond to the storage, transmission, and computational challenges, the data are compressed up to the acceptable quality by applying complex and demanding algorithms for data compression.

Discrete-time signals can be transformed into other domains using different signal transformations. A few signals that have a dense representation in one domain could be sparse in some domain such as time, frequency or space. A signal, which has a few nonzero coefficients, is called a sparse signal or its transform in another domain is sparse [2,3].

CS is a relatively new scheme of data sampling technique, which use random sensing to sample the signal that is spares in one of its transform domain. CS allows recovering sparse signal from a reduced set of observations. A reduced set of observations can be a result of a desire to sense a sparse signal with as low as possible number of measurements/observations [4-6].

The CS technique is widely utilized in the practical applications such as speech enhancement, radar, wireless communication, 
image processing, and observing the electrical activities of the human body [7, 8].

Recent results have completely demonstrated that the performance of all CS reconstruction algorithms depends on numerous parameters, for example, Sparsity level, level of under-sampling, measurement noise power, and the statistical distribution of non-zero elements of a sparse signal [9].

In this work, the CS scheme is applied on four types of testing signals. These can cover a wide range of applications. For every signal, three transformation techniques are utilized to sparsify the interested signal. The performance of the proposed system with different transform domains is assessed using root mean square error (RMSE) and the time taken by the reconstruction process to recover the signal.
In the second part of this study, the testing signals are corrupted by AWG $\mathrm{N}$ and the CS system is used as a denoising scheme. In addition, the universal threshold is modified for trimming the DCT coefficients. The system performance in this time is evaluated using $\mathrm{SNR}_{\mathrm{o}}$ for each case.

The remaining of this paper is organized in the following way: The second section, provides the Fundamentals principles of the CS. The third Section, deals with the proposed system. Simulation results with conclusions are given in sections 4 and 5 respectively.

\section{2- Fundamentals of CS}

The CS system can be implemented through two phases. These are the sampling/sensing phase and the reconstruction phase. Figure (1) shows the schematic diagram of the CS system. The two phases are explained in the next sub-sections.

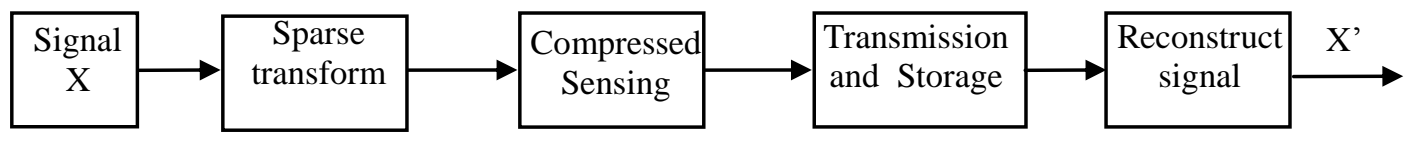

Fig. (1):- The Schematic Diagram of the CS System [10]

\section{2-1 Sensing Phase}

Assume that $\mathrm{x}(\mathrm{n})$ is a real-valued, discretetime signal of limited length. It can be viewed as a $\mathrm{N} \times 1$ column vector $\mathrm{x}$ with constituents $\mathrm{x}_{\mathrm{i}}, \mathrm{i}=1$, ..., $N$. The signal $x$ can be expressed as

$$
\mathbf{x}=\boldsymbol{\Psi} \mathbf{f}
$$

Where $\Psi$ is an $\mathrm{N} \times \mathrm{N}$ orthonormal basis matrix and $\mathbf{f}$ is the vector of coefficients, a $\mathrm{N} \times 1$ column vector. $\mathbf{x}$ and $\mathbf{f}$ are descriptions of the signal in different domains. Here, the time and the frequency domains are considered. Familiar basis or representations are DFT, DCT, and DWT.

The signal $\mathbf{x}$ is considered as a $K$-sparse function, if $\mathbf{f}$ has $\mathrm{K}$ significant and $(\mathrm{N}-\mathrm{K})$ nonsignificant coefficients. In general, $\mathbf{x}$ is compressible if the coefficient vector has a few large elements, while the remaining elements are small.

Based on what mentioned above, in this part we can clarify whether one can discard an extensive portion of the coefficients $f_{i}$ without much loss in x or not. Accordingly, we consider $\boldsymbol{x}_{\tau}=\boldsymbol{\Psi} \boldsymbol{f}_{\tau}$

Where $x_{\tau}$ is the vector of coefficients $x_{i}$ with all but the K-largest coefficients set to zero. In other words, after the thresholding process the signal can be represented by a linear combination of $\mathrm{K}(\mathrm{K}<<\mathrm{N})$ basis function.

Now consider a measurement of the signal $\mathrm{x}$ which results in the vector y having $\mathrm{M}<\mathrm{N}$ elements, representing the $\mathrm{M}$

measurements. This is characterized by

$$
\mathbf{y}=\boldsymbol{\Phi}
$$

with the $\mathrm{M}<<\mathrm{N}$ measurement matrix. In the case of random sampling, $\Phi$ is composed of $\mathrm{M}$ rows of a $\mathrm{N} \times \mathrm{N}$ identity matrix. This corresponds to selecting $\mathrm{M}$ out of $\mathrm{N}$ samples of $\mathrm{x}$. Substituting Eq. (1) into Eq.(3) we have

$$
\mathbf{y}=\boldsymbol{\Phi} \Psi \mathbf{f}=\boldsymbol{\Theta f}
$$

where the $M \times N$ matrix $\Theta$ is called the sensing matrix or sampling matrix[11].

For successful compressive sensing, suitable sensing matrix is required. Some of the most used sensing matrices include matrix randomly sampled from Fourier transform or Walsh-Hadamard transform, random matrix, Toeplitz matrix, Circulant matrix, random demodulation matrix, 
Gaussian or Bernoulli matrix, deterministic matrix, random convolution matrix, and DCT [12].

In this paper, the random Gaussian matrix is utilized to sample the input signal. Random Gaussian matrix is opted because it is simple, universal and easy to implement. Universality means that the signal can be sparse in any domain or any basis.

\section{2-2 Reconstruction Phase}

Once CS based sampling is performed, producing a sub-sampled signal $y$, it becomes the responsibility of the CS recovery algorithm to produce an estimate of the input signal $x$. There are various algorithms developed specifically for CS recovery, and can be classified into one of the following: greedy methods, convex-optimization techniques and combinatorial methods. The CS recovery algorithms, all sparse recovery methods must optimize the following.

- Minimal number of measurements.

- Robustness to noise and mismatch errors.

- Speed of computation.

- Performance guarantee on recovery.

Equation (4) is an underdetermined system of $\mathrm{M}$ equations with $\mathrm{N}$ unknowns, the $\mathrm{N}$ elements of $f$. However, if $f$. is sparse, CS theory shows that it is possible to find a solution if $\boldsymbol{\Theta}$ satisfies the restricted isometry property RIP [13]. A vector that minimizes the $\mathrm{L} 1$ norm gives the solution.

$$
\left\|f^{\prime}\right\|_{1}=\sum_{i=1}^{N}\left|f_{i}^{\prime}\right|
$$

That is from all vectors $f^{\prime}$ satisfying $\boldsymbol{\Theta} f^{\prime}=\mathbf{y}$ we select the one with minimum $l 1$ norm. Once we know $\hat{\boldsymbol{f}}$, we can reconstruct the signal

$$
\mathbf{x}=\boldsymbol{\Psi} \hat{\boldsymbol{f}}
$$

Finding $\hat{\boldsymbol{f}}$ is an optimization problem, which can be solved in a linear program.

\section{2-3 RIP}

There are two essential conditions that should be met in order to accomplish successful reconstruction for a wider range of sparsity level. First, is the (RIP). For a proper isometry constant, RIP ensures that any subset of columns in $\boldsymbol{\Theta}$ with sparsity level less than $\mathrm{K}$, is approximately orthogonal. This will ensure the successful signal reconstruction from small set of measurements. The second condition is a small correlation between the transform representation matrix and the measurement matrix (incoherence property).

The random Gaussian measurement matrix satisfy the RIP. Each type of measurement matrix has a different lower bound for the number of measurements. Hence, in the case of Gaussian random matrix with zero mean and variance $1 / \mathrm{M}$, the lower bound:

$$
M \geq C . K \cdot \log \left(\frac{N}{K}\right)
$$

is achievable and can guarantee the exact reconstruction of $\mathrm{x}(\mathrm{C}$ is a positive constant).

\section{3- PROPOSED SYSTEM}

This paper considers four types of testing signals suggested by donoho and jonosten [14]. These signals are HeavySine, Blocks, Doppler and Bumps. Figure (2), shows the flowchart of the proposed system. It illustrates CS implementation steps. 


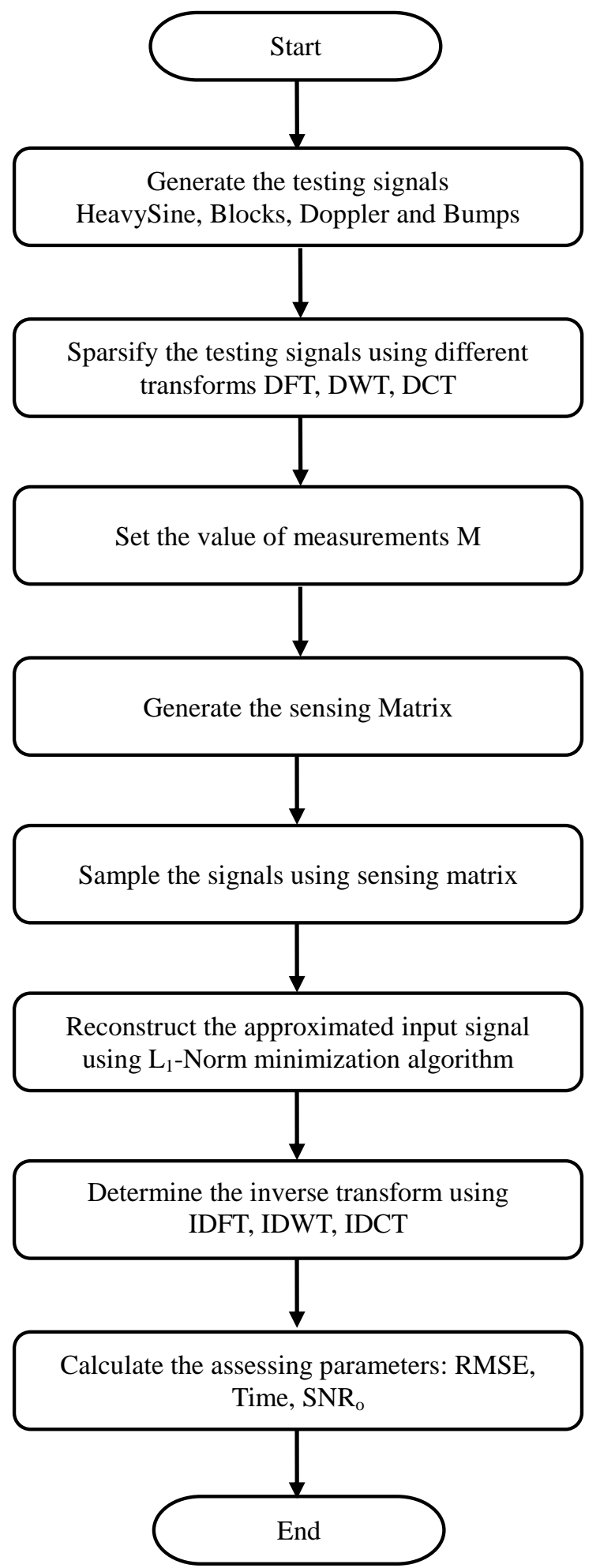

Fig. (2):- the flowchart of the proposed system

At first, the signals are generated. These signals are dense in time domain but they can be sparse in the proper basis. The signals are sparsified using different transforms. The transforms are DFT, DWT and DCT. Once, the signal is transformed to a new domain in which 
the signal is sparse, some elements must be pruned by setting different values for $M$ (number of observations). After that, the sensing matrix is generated and the testing signals are sampled using compressive sampling.

In the case of corrupted signals by AWGN, a hard threshold is applied. The elements that are less than the threshold value $\gamma$ are deleted and the number of measurements $M$ is adjusted by computing the non-zero elements.

$$
\gamma=\sigma(2 \ln (N))^{\frac{1}{2}}
$$

$$
\sigma=\frac{\operatorname{Median}(|c|)}{0.6745}
$$

Where $\mathrm{N}$ is the signal length, $\sigma$ is the standard deviation of zero mean AWGN, estimated by Donoho and Johnoston, and $\mathrm{c}$ is the detail coefficient of DWT. The FFT and DWT coefficients are pruned using equation (8).

In order to suggest a threshold value for the DCT coefficients, we have to recall the equation of the DCT, which is given in equation (10)[15].

$$
\begin{gathered}
X_{D C T}(k)=\sqrt{\frac{2}{N}} c(k) \sum_{n=0}^{N-1} x(n) \cos \left[\frac{(2 n+1) k \pi}{2 N}\right] \quad k \\
=0,1, \ldots \ldots N-1
\end{gathered}
$$

It can be noticed that the coefficients of the DCT are scaled by $\sqrt{\frac{2}{N}}$, thus we suggest to use $\gamma$ as in equation (8) but after scaling it by $\sqrt{\frac{2}{N}}$ as given in equation (11).

$\gamma_{D C T}=\sqrt{\frac{2}{N}} \gamma$

At this point, the $\mathrm{L}_{1}$ - Norm minimization algorithm is utilized to reconstruct the approximated input signals. Then the inverse transform is determined using IDFT, IDWT and IDCT.

With the completion of these steps, the assessing parameters for each reconstructed signal are calculated.

In this work, the following evaluation factors are used:

a- Root Mean Square Error (RMSE) is defined as

$R M S E=\left(\frac{1}{N} \sum_{n=1}^{N}[x(n)-\hat{x}(n)]^{2}\right)^{\frac{1}{2}}$

Where $x(n)$ and $\hat{x}(n)$ are the original and reconstructed (estimated) signals respectively.

\section{b- Output SNR}

$S N R_{o}=10 \log \left[\frac{\sum_{n=1}^{N} x(n)^{2}}{\sum_{n=1}^{N}|x(n)-\hat{x}(n)|^{2}}\right] \ldots \ldots$ c- Time taken by the optimization function to estimate the input signal. This time is determined using MATLAB script.

\section{4- Simulation Results}

This section describes the simulation of the proposed system with its results using MATLAB software package. A MATLAB script is written to examine the ability of the proposed scheme in recovering the testing signals from compressed samples. The first step in this process was to generate the four testing signals Blocks, Bumps, HeavySine, and Doppler using wnoise() function. Each signal is characterized by 2048 samples. Prior to the compressive sampling process, the four signals are preprocessed using different transformation techniques which are FFT, DCT and DWT, and consequently, the four signals are sparsified. After converting the testing signals from dense domain to sparse domains, the random Gaussian sensing matrix is created. On the completion of sensing matrix generation, the signals are compressively sampled through multiplying the sensing matrix by testing signals. Following this treatment, the testing signals are recovered by utilizing a free software package called L1-Magic [16]. At the finale, the signals are re-backed to time domain using IFFT, IDCT and IDWT functions. The results of this work are average values over 5 runs for the four testing signals.

In the simulation part, two experiments are executed. At first the effect of number of 
measurements or observations on the RMSE for each reconstructed signal are obtained. The Tables $1,2,3$ and 4 display the RMSE values of four tested signals with different values of measurements $M$ and different transform. The DWT is implemented using two families, which are daubechies 1 and coiflets 1 because they are widely used in signal denoising. In the case of CS with DWT, the CS is applied individually on approximation coefficients (CA) and details coefficients (CD). The RMSE values of the reconstructed signals using CS with DWT applied on CA are not acceptable thus, it is not recommended to apply CS on CA of the DWT because most of these coefficients are significant values. As can be seen from the above tables, the CS with DWT applied on CD is outperform CS with DCT and CS with FFT, for the four testing signals. As a comparison between CS with DCT and CS with FFT, CS with DCT performs better than CS with FFT. Figure (3), Figure (4), Figure (5) and Figure (6), display the reconstructed Doppler, HeavySine, Blocks and Bumps signals, which are sampled by a sensing matrix of size $(\mathrm{M} \times \mathrm{N}),(\mathrm{M}=1024, \mathrm{~N}=2048)$ thus the signals are reconstructed after deleting half of the samples.

Table (1):-RMSE Values of the Reconstructed Blocks Signal for different values of M

\begin{tabular}{ccccccc}
\hline $\begin{array}{c}\text { Measurements } \\
\mathbf{M}\end{array}$ & DCT & FFT & $\begin{array}{c}\text { DWT } \\
\text { DB1 CA }\end{array}$ & $\begin{array}{c}\text { DWT DB1 } \\
\text { CD }\end{array}$ & $\begin{array}{c}\text { DWT } \\
\text { Coif1 } \\
\text { CA }\end{array}$ & $\begin{array}{c}\text { DWT Coif1 } \\
\text { CD }\end{array}$ \\
\hline 400 & 0.7340 & 1.4710 & 4.5373 & $3.2861 \mathrm{e}-7$ & 4.4693 & $1.1796 \mathrm{e}-6$ \\
\hline 800 & 0.4083 & 0.6734 & 4.1350 & $1.5225 \mathrm{e}-7$ & 3.9809 & $1.1363 \mathrm{e}-7$ \\
\hline 1200 & 0.2510 & 0.4785 & 3.4781 & $4.3511 \mathrm{e}-8$ & 3.3887 & $3.359 \mathrm{e}-8$ \\
\hline 1600 & 0.1555 & 0.1671 & 2.5729 & $4.1980 \mathrm{e}-8$ & 2.5208 & $3.4376 \mathrm{e}-8$ \\
\hline 2000 & 0.0454 & 0.0353 & 0.8930 & $1.1672 \mathrm{e}-8$ & 0.9205 & $5.2908 \mathrm{e}-9$ \\
\hline
\end{tabular}

Table (2):- RMSE Values of the Reconstructed Bumps Signal for different values of M

\begin{tabular}{|c|c|c|c|c|c|c|}
\hline $\begin{array}{c}\text { Measurements } \\
\text { M }\end{array}$ & $\overline{D C T}$ & $\overline{\text { FFT }}$ & $\begin{array}{c}\text { DWT DB1 } \\
\text { CA }\end{array}$ & $\begin{array}{c}\text { DWT DB1 } \\
\text { CD }\end{array}$ & $\begin{array}{c}\text { DWT Coif1 } \\
\text { CA }\end{array}$ & $\begin{array}{c}\text { DWT Coif1 } \\
\text { CD }\end{array}$ \\
\hline 400 & 0.3738 & 0.9589 & 2.6067 & 0.0567 & 2.6059 & 0.0071 \\
\hline 800 & 0.0537 & 0.7029 & 2.1785 & 0.0287 & 2.1087 & 0.0034 \\
\hline 1200 & 0.0044 & 0.3028 & 1.0287 & 0.0124 & 1.2423 & 0.0014 \\
\hline 1600 & $1.3712 \mathrm{e}-4$ & 0.0580 & 0.1161 & 0.0014 & 0.0891 & $9.6451 e-5$ \\
\hline 2000 & $1.3296 \mathrm{e}-5$ & $3.2051 e-5$ & 0.0015 & $1.0521 \mathrm{e}-5$ & 0.0024 & $1.4689 \mathrm{e}-6$ \\
\hline
\end{tabular}

Table (3):- RMSE Values of the Reconstructed HeavySine Signal for different values of M

\begin{tabular}{ccccccc}
\hline $\begin{array}{c}\text { Measurements } \\
\text { M }\end{array}$ & DCT & FFT & $\begin{array}{c}\text { DWT DB1 } \\
\text { CA }\end{array}$ & $\begin{array}{c}\text { DWT DB1 } \\
\text { CD }\end{array}$ & $\begin{array}{c}\text { DWT Coif1 } \\
\text { CA }\end{array}$ & $\begin{array}{c}\text { DWT Coif1 } \\
\text { CD }\end{array}$ \\
\hline 400 & 0.1365 & 0.2183 & 4.7437 & 0.0130 & 4.5280 & $6.5989 \mathrm{e}-5$ \\
\hline 800 & 0.0852 & 0.1492 & 4.2143 & 0.0122 & 4.2324 & $6.1338 \mathrm{e}-5$ \\
\hline 1200 & 0.0558 & 0.0866 & 3.5825 & 0.0107 & 3.6793 & $5.3381 \mathrm{e}-5$ \\
\hline 1600 & 0.0325 & 0.0440 & 2.7364 & 0.0092 & 2.8175 & $4.3605 \mathrm{e}-5$ \\
\hline 2000 & 0.0078 & 0.0079 & 0.8827 & 0.0043 & 0.9286 & $2.296 \mathrm{e}-5$ \\
\hline
\end{tabular}

Table (4):- RMSE Values of the Reconstructed Doppler Signal for different values of M

\begin{tabular}{|c|c|c|c|c|c|c|}
\hline $\begin{array}{c}\text { Measurements } \\
\mathrm{M}\end{array}$ & DCT & $\overline{F F T}$ & $\begin{array}{c}\text { DWT DB1 } \\
\text { CA }\end{array}$ & $\begin{array}{c}\text { DWT DB1 } \\
\text { CD }\end{array}$ & $\begin{array}{c}\text { DWT Coif1 } \\
\text { CA }\end{array}$ & $\begin{array}{l}\text { DWT Coif1 } \\
\text { CD }\end{array}$ \\
\hline 400 & 0.0864 & 0.2043 & 0.4276 & 0.0118 & 0.4268 & 0.0027 \\
\hline 800 & 0.0359 & 0.0779 & 0.4050 & 0.0080 & 0.4139 & $4.5295 \mathrm{e}-4$ \\
\hline 1200 & 0.0122 & 0.0489 & 0.3470 & 0.0048 & 0.3520 & $1.1297 \mathrm{e}-4$ \\
\hline 1600 & 0.0019 & 0.0184 & 0.2831 & 0.0024 & 0.2766 & $2.2186 \mathrm{e}-5$ \\
\hline 2000 & $1.905 e-5$ & $8.4336 e-5$ & 0.0978 & $5.3385 e-4$ & 0.1068 & $3.228 \mathrm{e}-6$ \\
\hline
\end{tabular}



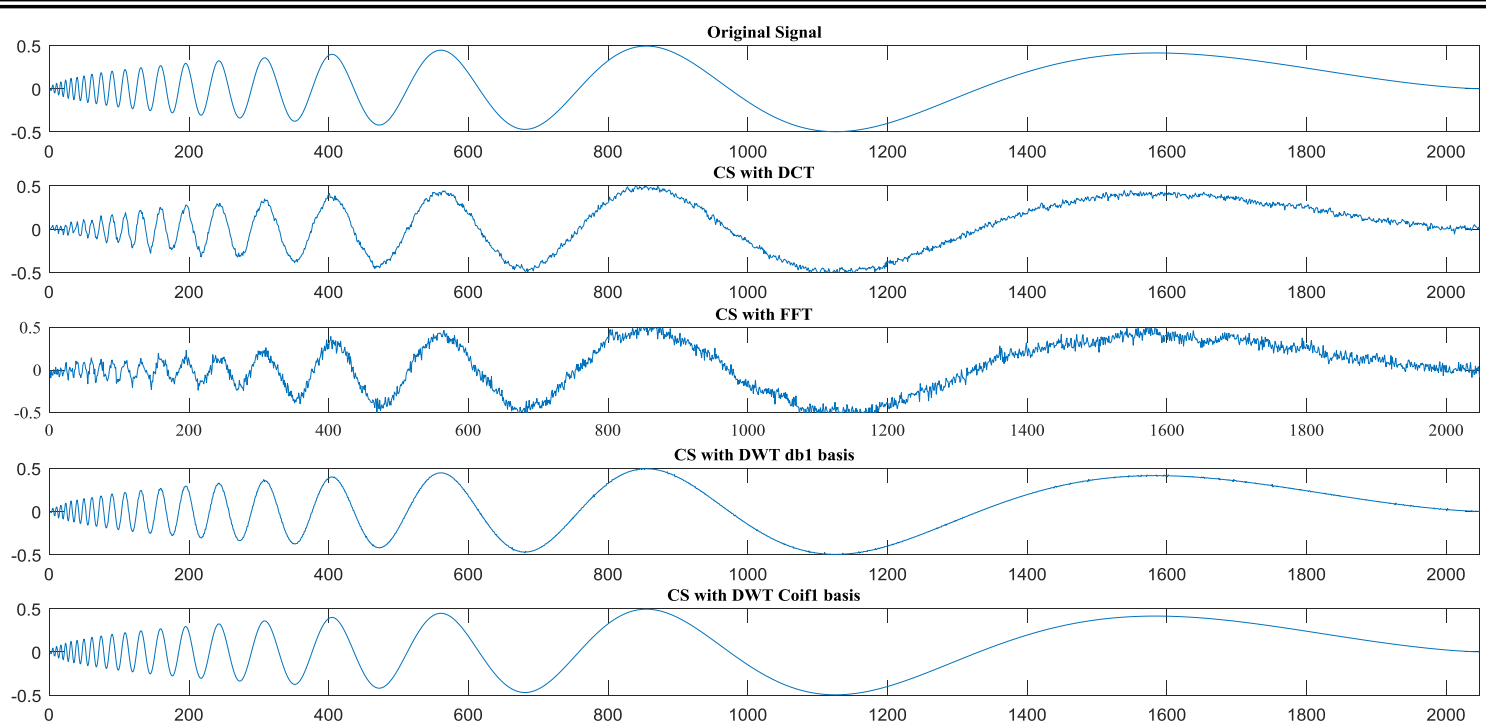

Figures (3), The reconstructed Doppler signal using CS with different transforms $(M=1024)$
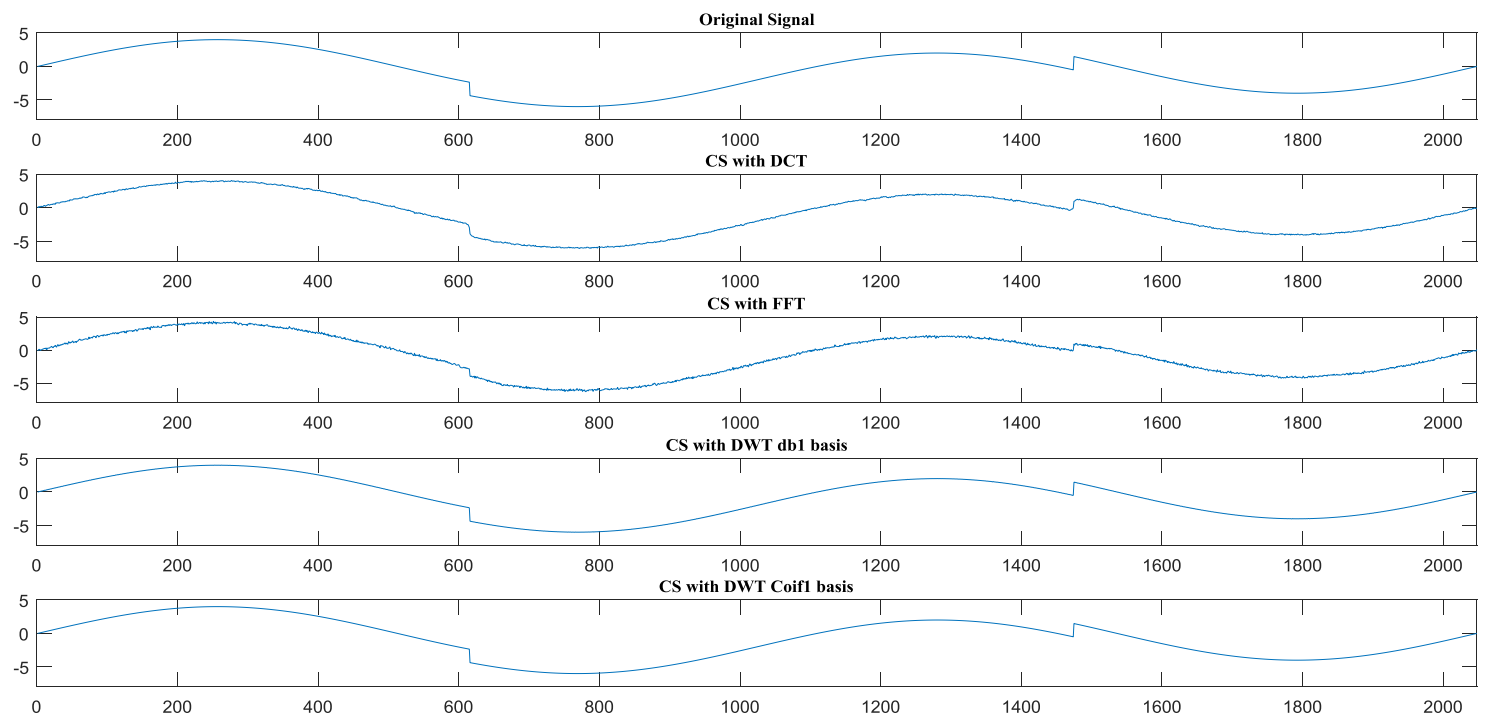

Figures (4), The reconstructed HeavySine signal using CS with different transforms (M=1024) 

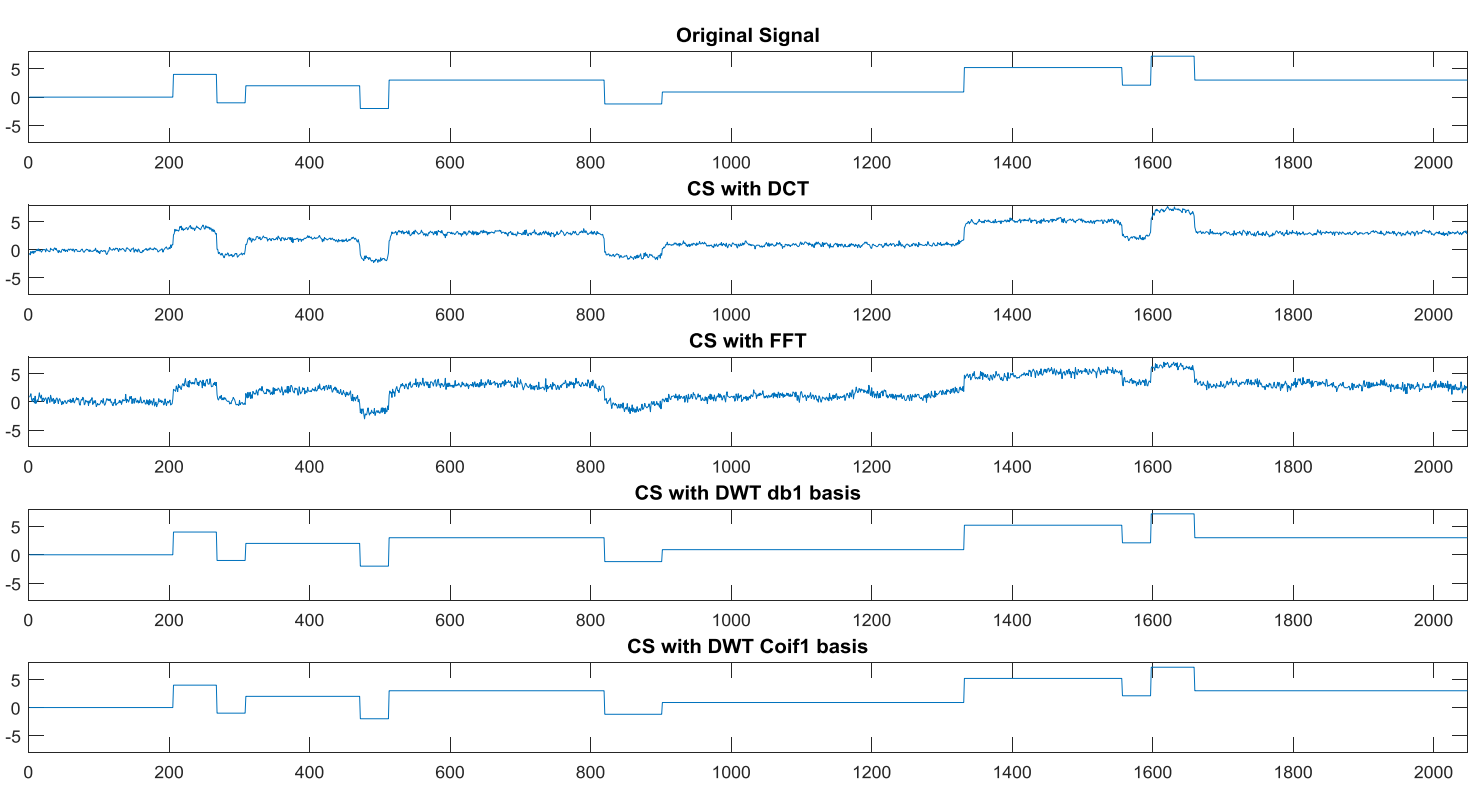

Figures (5), The reconstructed Blocks signal using CS with different transforms (M=1024)
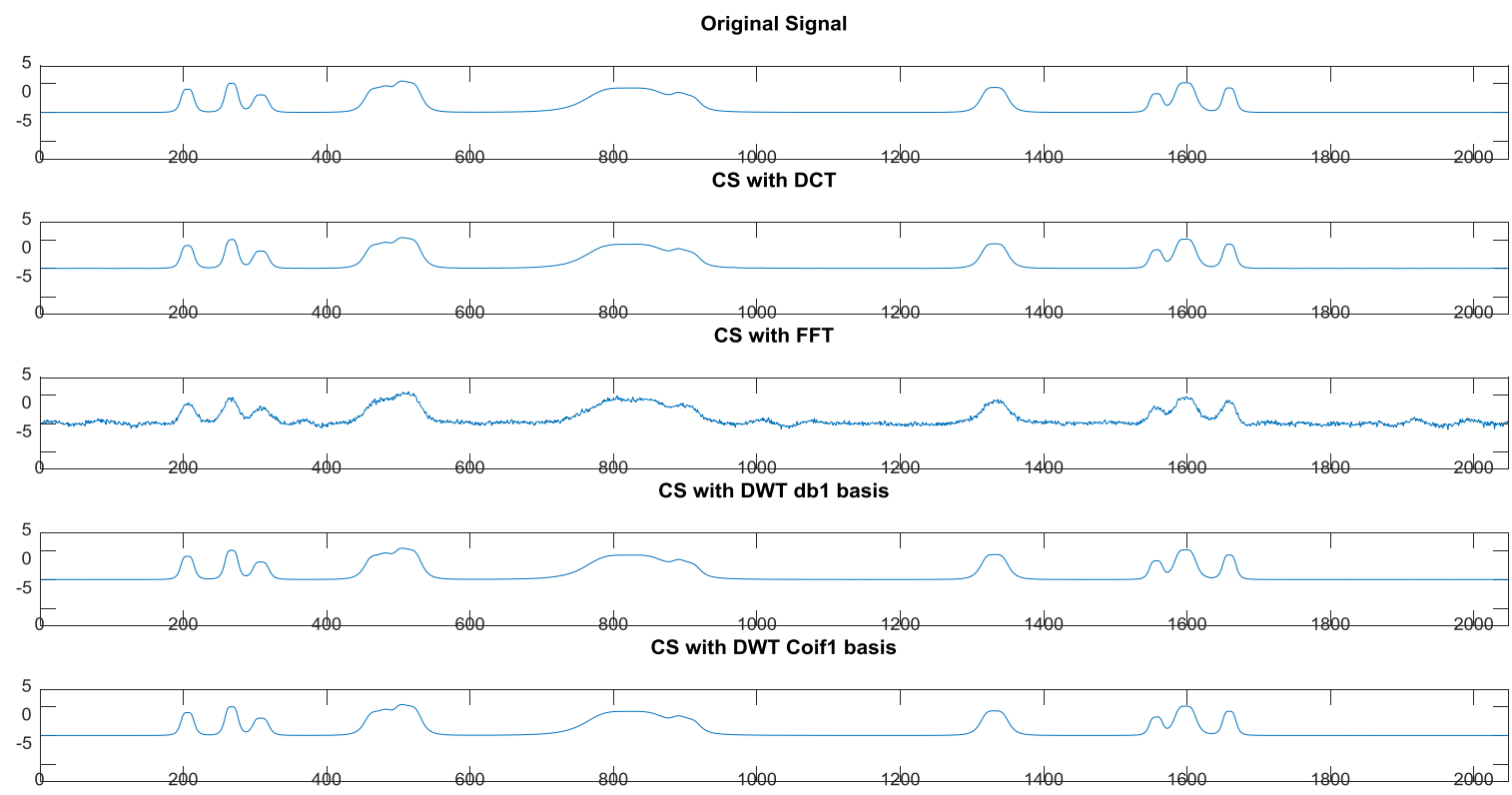

Figures (6), The reconstructed Bumps signal using CS with different transforms (M=1024) 
The tables 5, 6, 7 and 8 provide the time taken (in seconds) by the L1-magic function to reconstruct the four testing signals. Again, the CS with DWT outperforms the others.

Table (5):- Time taken (in seconds) by L1-magic to Reconstruct Blocks Signal for different values of M

\begin{tabular}{ccccccc}
\hline $\begin{array}{c}\text { Measurements } \\
\mathbf{M}\end{array}$ & DCT & FFT & DWT DB1 CA & DWT DB1 CD & $\begin{array}{c}\text { DWT Coif1 } \\
\text { CA }\end{array}$ & $\begin{array}{c}\text { DWT Coif1 } \\
\text { CD }\end{array}$ \\
\hline 400 & 2.445489 & 4.984162 & 0.565039 & 0.311339 & 0.653135 & 0.565727 \\
\hline 800 & 5.911202 & 8.305987 & 1.015677 & 0.4422887 & 1.088137 & 0.656686 \\
\hline 1200 & 12.001254 & 12.15079 & 1.803465 & 0.757201 & 1.895024 & 0.913502 \\
\hline 1600 & 21.400325 & 38.528599 & 2.863318 & 1.019992 & 3.131341 & 1.223524 \\
\hline 2000 & 28.1607 & 69.755147 & 3.419567 & 1.53494 & 3.597302 & 1.685283 \\
\hline
\end{tabular}

Table (6):-Time taken (in seconds) by L1-magic to Reconstruct Bumps Signal for different values of M

\begin{tabular}{cllcccc}
\hline $\begin{array}{c}\text { Measurements } \\
\mathbf{M}\end{array}$ & DCT & FFT & DWT DB1 CA & DWT DB1 CD & $\begin{array}{c}\text { DWT Coif1 } \\
\text { CA }\end{array}$ & $\begin{array}{c}\text { DWT Coif1 } \\
\text { CD }\end{array}$ \\
\hline 400 & 2.616347 & 4.706586 & 0.629739 & 0.539427 & 0.591839 & 0.517094 \\
\hline 800 & 7.281437 & 8.199710 & 1.016012 & 1.001984 & 1.056784 & 0.95074 \\
\hline 1200 & 15.066650 & 27.935582 & 1.911401 & 1.854934 & 1.789974 & 1.635013 \\
\hline 1600 & 24.932513 & 88.882971 & 3.34314 & 3.022442 & 3.59434 & 2.722893 \\
\hline 2000 & 18.264106 & 131.623723 & 5.261913 & 4.960288 & 5.367138 & 2.967109 \\
\hline
\end{tabular}

Table (7):- Time taken (in seconds) by L1-magic to Reconstruct HeavySine Signal for different values of $\mathrm{M}$

\begin{tabular}{cllcccc}
\hline $\begin{array}{c}\text { Measurements } \\
\mathbf{M}\end{array}$ & DCT & FFT & DWT DB1 CA & DWT DB1 CD & $\begin{array}{c}\text { DWT Coif1 } \\
\text { CA }\end{array}$ & $\begin{array}{c}\text { DWT Coif1 } \\
\text { CD }\end{array}$ \\
\hline 400 & 2.692652 & 4.125602 & 0.580958 & 0.585103 & 0.600505 & 0.56922 \\
\hline 800 & 6.385464 & 11.232797 & 1.399249 & 0.986099 & 1.057430 & 0.810918 \\
\hline 1200 & 11.975623 & 24.074669 & 1.959287 & 1.500744 & 1.770792 & 1.226418 \\
\hline 1600 & 19.683648 & 98.402756 & 2.817492 & 2.433053 & 2.860797 & 1.849281 \\
\hline 2000 & 28.939284 & 157.121291 & 7.119102 & 3.290138 & 3.208479 & 2.706948 \\
\hline
\end{tabular}

Table (8):- Time taken (in seconds) by L1-magic to Reconstruct Doppler Signal for different values of M

\begin{tabular}{lllllll}
$\begin{array}{c}\text { Measurements } \\
\mathbf{M}\end{array}$ & DCT & FFT & DWT DB1 CA & DWT DB1 CD & $\begin{array}{c}\text { DWT Coif1 } \\
\text { CA }\end{array}$ & $\begin{array}{c}\text { DWT Coif1 } \\
\text { CD }\end{array}$ \\
\hline 400 & 2.581048 & 1.749504 & 0.505288 & 0.540821 & 0.518549 & 0.544389 \\
\hline 800 & 6.635624 & 8.780828 & 1.005269 & 0.969398 & 1.044102 & 0.987540 \\
\hline 1200 & 12.632409 & 14.985458 & 1.731112 & 1.4585 & 1.803332 & 1.595637 \\
\hline 1600 & 25.284833 & 77.059023 & 2.617687 & 2.326997 & 2.771761 & 2.368390 \\
\hline 2000 & 27.41301 & 115.93344 & 3.09954 & 2.680331 & 3.160513 & 2.724257 \\
\hline
\end{tabular}

Secondly, the four testing signals are corrupted by AWGN then; the CS scheme is utilized as a denoising technique. The universal threshold as explained section 3 is used in pruning the elements of the sparse signals. Table (9) gives the results of denoising Blocks signal, it can be seen that CS with FFT and DCT achieves enhancement of $3 \mathrm{~dB}$, on the other hand CS with DWT of two basis and for single decomposition level accomplishes enhancement of $5 \mathrm{~dB}$.
Table (10) provides the result of denoising Bumps signal, CS with FFT and DCT improve the signal by $1 \mathrm{~dB}$ while CS with DWT of DB1 and Coif 1 basis improves the signal by $4 \mathrm{~dB}$. Tables 11 and 12 present the results of Heavy Sine and Doppler signals; it can be noticed that CS with DWT reduces the noise by $3 \mathrm{~dB}$ but no increase in SNR is obtained using CS with DCT and FFT. 
Table(9):- Results of denoising the Blocks signal using CS with different transforms

\begin{tabular}{lllll}
\hline & \multicolumn{4}{c}{$\mathbf{S N R}_{\mathbf{0}} \mathbf{d B}$} \\
\hline $\begin{array}{l}\mathrm{SNR} \\
\mathrm{dB}\end{array}$ & FFT & DCT & $\begin{array}{l}\text { DWT } \\
\text { DB1 }\end{array}$ & $\begin{array}{l}\text { DWT } \\
\text { Coif1 }\end{array}$ \\
\hline 0 & 3.61 & 3.58 & 6.3728 & 6.6655 \\
\hline 5 & 8.4 & 8.45 & 11.2586 & 11.2517 \\
\hline 10 & 13.58 & 13.55 & 15.5946 & 16.2240 \\
\hline 15 & 18.56 & 18.5 & 19.4242 & 20.2238 \\
\hline
\end{tabular}

Table( 10):- Results of denoising the Bumps signal using CS with different transforms

\begin{tabular}{lllll}
\hline & \multicolumn{4}{c}{$\mathbf{S N R}_{\mathbf{0}} \mathbf{d B}$} \\
\hline $\begin{array}{l}\text { SNR in } \\
\mathrm{dB}\end{array}$ & FFT & DCT & $\begin{array}{l}\text { DWT } \\
\text { DB1 }\end{array}$ & $\begin{array}{l}\text { DWT } \\
\text { Coif1 }\end{array}$ \\
\hline 0 & 1.17 & 1.435 & 4.2765 & 4.3327 \\
\hline 5 & 6.38 & 6.423 & 9.5884 & 9.3216 \\
\hline 10 & 11.26 & 11.3 & 14.2637 & 14.3943 \\
\hline 15 & 16.36 & 16.28 & 19.1724 & 19.4732 \\
\hline
\end{tabular}

Table( 11):- Results of denoising the Heavy Sine signal using CS with different transforms

\begin{tabular}{lllll}
\hline & \multicolumn{4}{c}{$\mathbf{S N R}_{\mathbf{0}} \mathbf{d B}$} \\
\hline $\begin{array}{l}\text { SNR in } \\
\text { dB }\end{array}$ & FFT & DCT & $\begin{array}{l}\text { DWT } \\
\text { DB1 }\end{array}$ & $\begin{array}{l}\text { DWT } \\
\text { Coif1 }\end{array}$ \\
\hline 0 & 0.35 & 0.57 & 3.4433 & 3.6359 \\
\hline 5 & 5.33 & 5.521 & 8.5082 & 8.0830 \\
\hline 10 & 10.24 & 10.33 & 13.2922 & 13.4120 \\
\hline 15 & 15.54 & 15.35 & 18.2093 & 18.3390 \\
\hline
\end{tabular}

Table (12):-, Results of denoising the Doppler signal using CS with different transforms

\begin{tabular}{lllll}
\hline & \multicolumn{4}{c}{$\mathbf{S N R}_{\mathbf{0}} \mathbf{d B}$} \\
\hline $\begin{array}{l}\mathrm{SNR}_{\text {in }} \\
\mathrm{dB}\end{array}$ & FFT & DCT & $\begin{array}{l}\text { DWT } \\
\text { DB1 }\end{array}$ & $\begin{array}{l}\text { DWT } \\
\text { Coif1 }\end{array}$ \\
\hline 0 & 0 & 0.302 & 3.2966 & 3.2387 \\
\hline 5 & 5.18 & 5.12 & 7.8665 & 8.3022 \\
\hline 10 & 10.1 & 10.127 & 12.9403 & 13.0624 \\
\hline 15 & 15.1 & 15.11 & 17.7866 & 18.1480 \\
\hline
\end{tabular}

Figures7,8,9 and 10 display the relationship between input SNR and output SNR of the signals blocks, Bumps ,Heavy Sine and Doppler after they are reconstructed by CS with different transforms.
These figures have revealed that the relation between input SNR and output SNR is linear for the proposed system. 


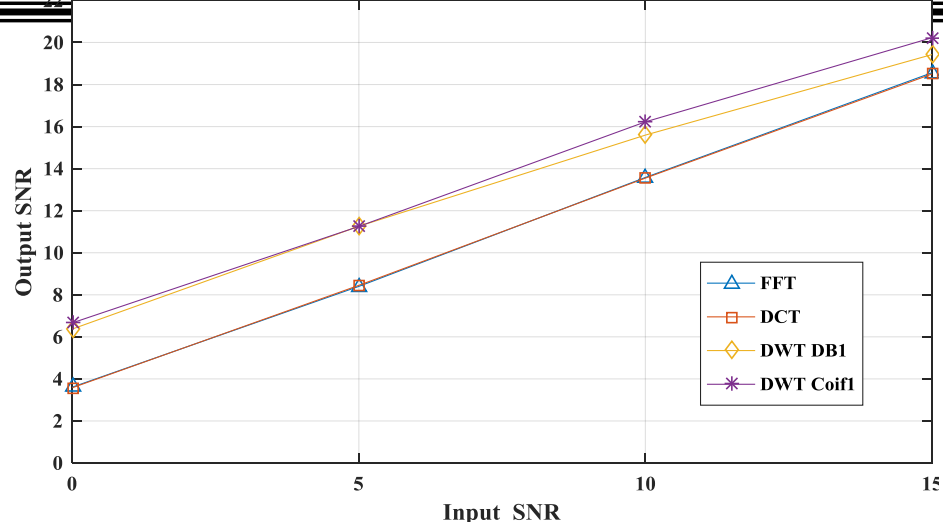

Fig.(7):- $\mathrm{SNR}_{\mathrm{o}}$ versus $\mathrm{SNR}_{\text {in }}$ for the reconstructed Blocks signal

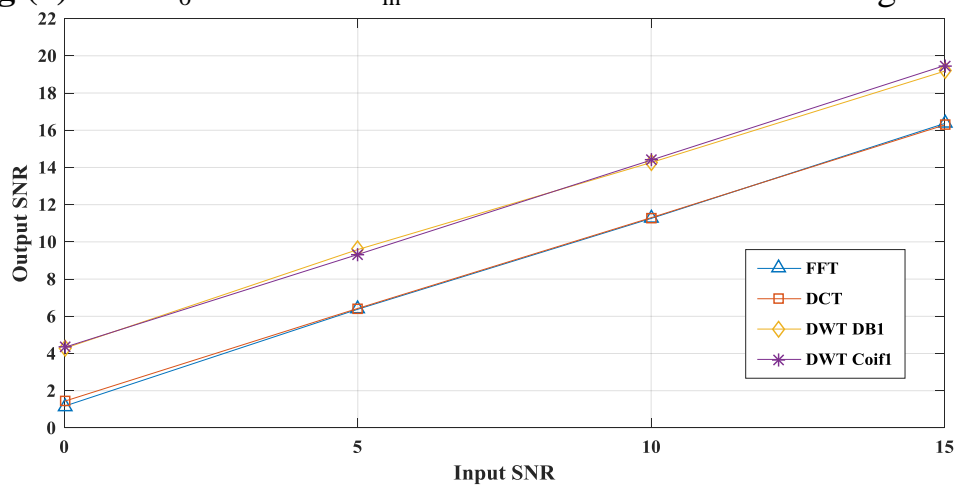

Fig.(8):- $\mathrm{SNR}_{\mathrm{o}}$ versus $\mathrm{SNR}_{\text {in }}$ for the reconstructed Bumps signal

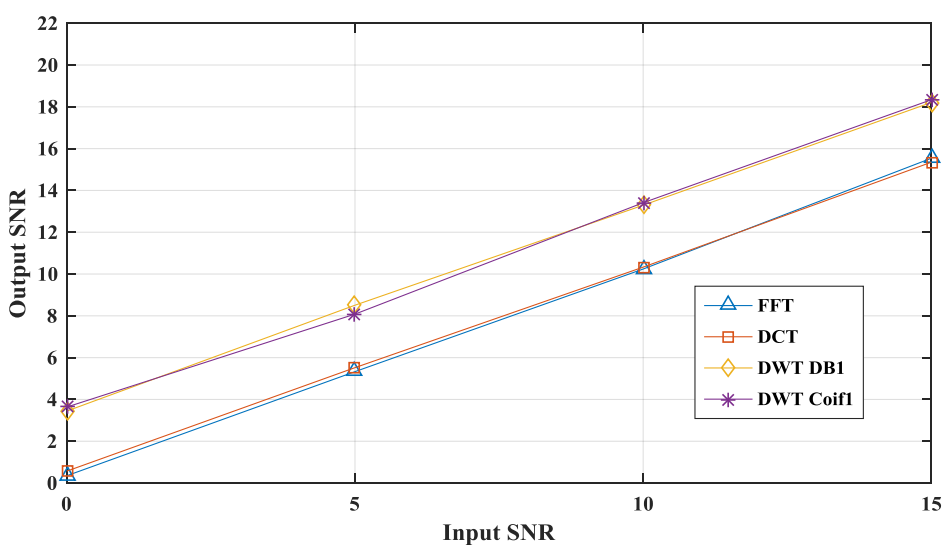

Fig. (9):- $\mathrm{SNR}_{\mathrm{o}}$ versus $\mathrm{SNR}_{\text {in }}$ for the reconstructed Heavy Sine signal 


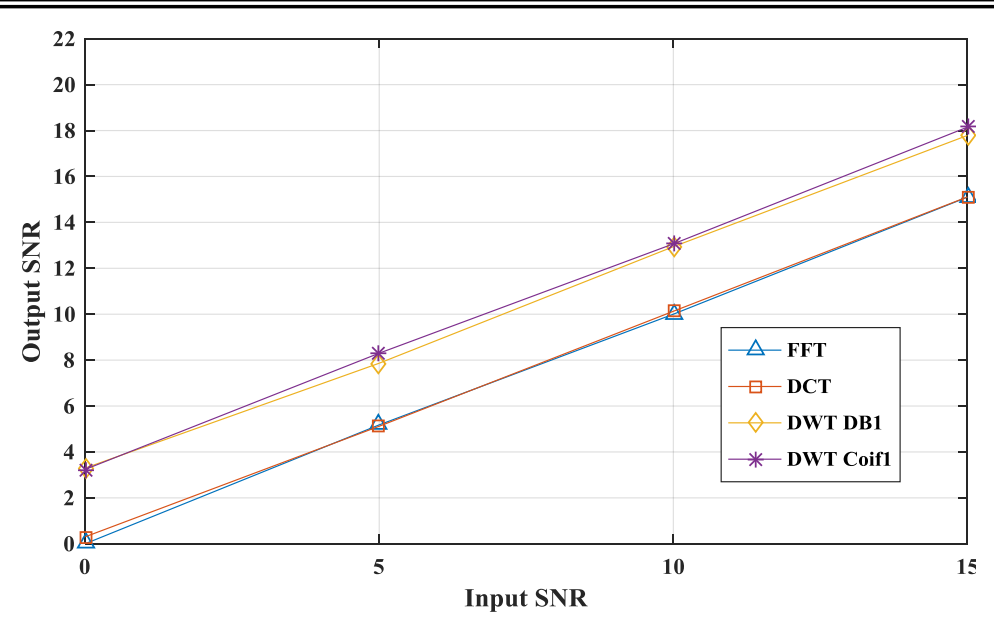

Fig. (10):- $\mathrm{SNR}_{\mathrm{o}}$ versus $\mathrm{SNR}_{\mathrm{in}}$ for the reconstructed Doppler signal

\section{5- CONCLUSIONS}

In this investigation, a CS with different signal transformation is proposed and simulated. Four types of testing signals, which are Blocks, Bumps, Heavy Sine and Doppler, are used. Random Gaussian matrix is selected because it is simple, universal and easy to implement. Furthermore, a modified universal threshold is proposed for pruning the coefficients of the DCT. This paper compares the impact of DFT, DCT and DWT transformations on CS Scheme. Three parameters that are RMSE, $\mathrm{SNR}_{0}$, and time to reconstruct the signals are employed to evaluate the performance of the proposed system. In addition, this study contributes to our understanding of CS technique. Simulation results have led us to conclude the followings:

- RMSE is inversely proportioned to the number of measurements/observations (M).

- Time taken to reconstruct the signal is directly proportioned to number of measurements (M).

- The relationship between $\mathrm{SNR}_{\mathrm{o}}$ and $\mathrm{SNR}_{\text {in }}$ is linear for the proposed system.

- CS with DWT outperforms the DCT and FFT in RMSE, reconstruction time, and $\mathrm{SNR}_{0}$ so we strongly recommend CS with DWT over DCT and FFT transformations.

- The proposed system has achieved less amount of SNR enhancement in the cases of Heavy Sine and Doppler signals so these type of signals require more processing.

- It is not recommended to apply CS on approximation coefficients (CA) of the DWT because most of these coefficients are significant (large values).

\section{6- REFERENCES}

1- Srdjan Stankovic', Irena Orovic', Ervin Sejdic (2016). Multimedia Signals and Systems (2 ${ }^{\text {nd }}$ ed.). Switzerland: Springer international Publishing.

2- Marvasti F. et al. (2012). A unified approach to sparse signal processing. EURASIP Journal on Advances in Signal Processing. From http://asp.eurasipjournals.com/content/2012/1/44.

3- Ljubisa Stankovic (2015). Digital Signal Processing: with selected topics. South Carolina, USA. CreateSpace independent Publishing Platform.

4- Xu Hong-kui, Jiang Ming-yan, Sun Wei-feng (2010). Compression Method for Non-Stationary Signals Based on Compressive Sensing. The Journal of China Universities of Posts and Telecommunications. From

www.sciencedirect.com/science/journal/10058885.

5- Emmanuel J. Candès, Michael B. Wakin (2008). An Introduction to Compressive Sampling. IEEE SIGNAL PROCESSING MAGAZINE. March 2008. (pp. 21-30).

6- Vivek K Goyal, Alyson K. Fletcher, Sundeep Rangan (2008). Compressive Sampling and Lossy Compression. IEEE SIGNAL PROCESSING MAGAZINE. March 2008. (pp. 48-56).

7- Mohammad Fardad, Sayed Masoud Sayedi (2016). A Low Complexity Hardware for Compressive Sensing Matrix Generation. 24th Iranian Conference on Electrical Engineering (ICEE). (pp. 638-642). Isfahan, Iran.

8- Pooja C. Nahar, Dr.Mahesh T. Kolte (2014). An Introduction to Compressive Sensing and its Applications. International Journal of Scientific and Research Publications. Volume 4, Issue 6, June 2014. 
9- Ahmed Zaki, Saikat Chatterjee, Lars K. Rasmussen (2015). Universal Algorithm for Compressive Sampling. 23 ${ }^{\text {rd }}$ European Signal Processing Conference (EUSIPCO). Sweden. (pp.689-693).

10- Lei Zhu; Yaolin Zhu; Huan Mao; Meihua Gu(2009).A New Method for Sparse Signal Denoising Based on Compressed Sensing. Second International Symposium on Knowledge Acquisition and Modeling. 2009, Volume: 1

11- Fereshteh Fakhar Firouzeh, Seyed Ghorshi, Sina Salsabili. (2014). Compressed Sensing based Speech Enhancement. 2014 8th International Conference on Signal Processing and Communication Systems (ICSPCS).

12- Fatima Salahdine, Naima Kaaboucha, Hassan El Ghazi.(2016). A survey on compressive sensing techniques for cognitive radio networks. Available online at www.elsevier.com/locate/phycom.

13- Carsten Roppel, Martin Danz.(2014) Compressive sampling experiments. 6th European Embedded Design in Education and Research Conference (EDERC). 2014

14- David L. Donoho, IAN M. Johnstone(1994). Adapting to Unknown Smoothness via Wavelet Shrinkage. Available online at statweb.stanford.edu/ imj/WEBLIST/1995/ausws.pdf.

15- Li Tan. (2013). Digital Signal Processing 2nd Edition fundamentals and applications. Elsevier Inc. 2013. Page 519.

16- Justin Romberg. L1-magic. Available online at : WWW.acm.caltech.edu/11 magic. 